\title{
A INSERÇÃO DOS PROFISSIONAIS INTEGRANTES DAS RESIDÊNCIAS MULTIPROFISSIONAIS EM SERVIÇOS DE SAÚDE: REVISÃO DE LITERATURA
}

\author{
THE INSERTION OF THE PROFESSIONAL MEMBERS OF MULTIDISCI- \\ PLINARY RESIDENCES IN HEALTH CARE: LITERATURE REVIEW
}

\author{
Janaína Lunardi Canabarro ${ }^{1}$, Daiana Foggiato de Siqueira ${ }^{2}$, \\ Marlene Gomes Terra ${ }^{3}$
}

Recebido em: 28/09/2016

Aprovado em: 01/12/2016

\begin{abstract}
RESUMO
O objetivo deste estudo consiste em identificar e analisar na produção científica os fatores que interferem na inserção dos profissionais integrantes das Residências Multiprofissionais em serviços de saúde. Para isso, realizou-se uma revisão de literatura na Base de Dados de Enfermagem (BDENF), na base de dados Literatura Latino-Americana e do Caribe em Ciências da Saúde (LILACS) e na Scientific Electronic Library Online (SciELO). O corpus desta revisão integrativa foi constituído de 14 artigos. A partir da análise do conteúdo dos artigos selecionados, emergiram as seguintes categorias: potencialidades da inserção dos profissionais integrantes da Residência Multiprofissional nos serviços; e fragilidades e necessidades para inserção dos profissionais integrantes da Residência Multiprofissional nos serviços de saúde. Diante dos resultados obtidos e da importância da Residência Multiprofissional para a formação profissional e para o serviço, é preciso pensar em estratégias para mudança de concepção quanto aos modelos paradigmáticos centrados na figura do biomédico, percebendo a necessidade das demais profissões.

Palavras-chave: Profissionais da Saúde; Residência não médica não odontológica; Pessoal de Saúde.
\end{abstract}

\begin{abstract}
The objective of this study is to identify and analyze in the scientific production the factors that interfere in the insertion of the professionals integrating the Multiprofessional Residences into health services. For that, an literature review was performed in the Nursing Database (BDENF), in the Latin American and Caribbean Literature in Health Sciences database (LILACS) and in the Scientific Electronic Library Online (SciELO). The corpus of this integrative review consisted of 14 articles. From the analysis of the content of the articles selected, the following categories emerged: potential of the insertion of the professionals integrating the Multiprofessional Residency into the services; And fragilities and needs for insertion of the professionals integrating the Multiprofessional Residency into the health services. In view of the results obtained and the importance of the Multiprofessional Residence for professional training and service, it is necessary to think about strategies to change conception regarding the paradigmatic models focused on the biomedical figure, realizing the need of the other professions.
\end{abstract}

Keywords: Health Professionals; not dental nonmedical residence; Health Personnel.

\footnotetext{
1 Enfermeira. Professora do curso técnico em enfermagem na Faculdade Integrada de Santa Maria. Mestranda do Programa de Pós Graduação em Enfermagem da Universidade Federal de Santa Maria (PPGEnf/UFSM). E-mail: jana.1.c2010@hotmail.com

2 Enfermeira. Mestra em Enfermagem. Doutoranda do Programa de Pós Graduação em Enfermagem da Universidade Federal de Santa Maria (PPGEnf/ UFSM). E-mail: daianasiqueira@yahoo.com.br

3 Enfermeira. Pós-doutora em Enfermagem pelo Programa de Pós-Graduação em Enfermagem da Universidade Federal do Rio Grande do Sul (UFRGS). Professora Associada da Universidade Federal de Santa Maria (UFSM), atuando na Graduação e Pós-Graduação em Enfermagem e na Residência Multiprofissional Integrada em Sistemas Públicos de Saúde. E-mail: martesm@hotmail.com.br
} 


\section{Introdução}

Frente às imprescindibilidades das práticas dos profissionais de saúde em responder às demandas do Sistema Único de Saúde (SUS), foram criados, entre outros programas, a Residência Multiprofissional em Saúde (RMS) como um dispositivo de transformação das práticas da área da saúde, fazendo parte da Política Nacional de Educação Permanente (PNEPS). Esta foi instituída pela Portaria n. ${ }^{\circ}$ 198, de 13 de fevereiro de 2004, em virtude da criação, em 2003, da Secretaria de Gestão do Trabalho e Educação na Saúde (SGTES) (BRASIL, 2006).

Ressalta-se que a RMS visa à formação de profissionais para uma atuação diferenciada no SUS, na perspectiva de mudanças na formação dos trabalhadores de saúde, bem como de incentivo à construção interdisciplinar, ao trabalho em equipe, à educação permanente em saúde e à reorientação das lógicas tecnoassistenciais (LOBATO, 2010). Desde a sua criação e regulamentação pela Lei Orgânica da Saúde n. ${ }^{\circ}$ 8080/90 e pela Lei Complementar n. ${ }^{\circ} 8142 / 90$, esse sistema vem enfrentando mudanças relacionadas às práticas de saúde, visando à reestruturação do modelo de saúde no Brasil. Assim, o Ministério da Saúde (MS) vem apoiando a RMS desde 2002, por meio do projeto ReforSUS (BRASIL, 1990, 1999).

Porém, essas medidas não têm sido suficientes para alcançar a qualidade desejada na saúde e para reestruturar o sistema no que concerne à formação profissional de trabalhadores com vistas a atingir a excelência na prestação do cuidado. Ainda que conquistas tenham ocorrido, a necessidade de avanço fazse presente em nossos cotidianos.

Desse modo, os profissionais integrantes da RMS precisam atuar em conjunto com as equipes de saúde e desenvolver ações de educação permanentes a favor da comunidade, sendo essas ações apoiadas nos princípios do
SUS. Diante do exposto, reconhecese o papel fundamental da RMS junto à PNEPS, política que possibilita mudanças na formação de trabalhadores condizentes com os princípios do SUS, por meio de um trabalho multiprofissional e em equipe que tem por objetivo o desenvolvimento de ações de educação permanente em saúde.

Nesse contexto, em 2005, foi instituída a Residência em Área Profissional da Saúde, definida como modalidade de ensino de pós-graduação lato sensu, voltada para a educação em serviço e destinada às categorias profissionais que integram a área de saúde, excetuando-se a médica, conforme artigo 13 da Lei n. ${ }^{\circ} 11.129 / 2005$. Diante disso, o objetivo deste estudo consiste em identificar e analisar na produção científica os fatores que interferem na inserção dos profissionais das Residências Multiprofissionais em serviços de saúde.

\section{Método de pesquisa}

Este estudo é uma revisão de literatura, com abordagem qualitativa, com a finalidade de reunir e sintetizar resultados de pesquisas sobre um tema ou uma questão de maneira sistemática e ordenada. Foram realizadas, para isso, algumas etapas da revisão: identificação do tema e seleção da hipótese ou questão de pesquisa; estabelecimento de critérios para inclusão e exclusão de estudos/amostragem ou busca na literatura; definição das informações a serem extraídas dos estudos selecionados/ categorização dos estudos; avaliação dos estudos incluídos na revisão integrativa; interpretação dos resultados; e apresentação da revisão/síntese do conhecimento (MENDES; SILVEIRA; GALVÃO, 2008).

O levantamento das publicações ocorreu na Base de Dados de Enfermagem (BDENF), sendo utilizada como estratégia de busca a palavra-chave "residência não médica não odontológica"; na base de dados Literatura Lati- 
no-Americana e do Caribe em Ciências da Saúde (LILACS), usando a palavrachave "residência" and "multiprofissional"; e na Scientific Electronic Library Online (SciELO), empregando a palavra-chave "residência multiprofissional". Os critérios de inclusão foram: artigos disponíveis na íntegra, on-line e de forma gratuita, escritos em língua portuguesa, inglesa ou espanhola e publicados no período entre 2005 e 2015 que respondessem ao objetivo deste estudo. O recorte temporal deve-se ao fato de a residência em área da saúde ter ser instituída a partir de 2005. Já os critérios de exclusão foram: teses e dissertações, estudos de revisão, reflexões e documentos ministeriais. O levantamento dos dados ocorreu em julho de 2016.

$\mathrm{Na}$ BDENF, foram encontrados 34 textos, dos quais 28 foram excluídos - 22 por terem sido publicados fora do recorte temporal estabelecido, dois por serem teses e três por não correspondem à temática pesquisada, restando apenas um artigo para compor o corpus da revisão. Na LILACS, foram encontrados 81 publicações, das quais 67 foram excluídas - 13 pelo critério temporal, 42 por não responderem à temática, uma por repetição na própria base de dados, uma por repetição na base de dados da BDENF, nove por serem teses, uma por ser documento ministerial e uma por ser resumo incompleto, restando 13 artigos para compor esta revisão. Já na SciELO, foram encontrados 35 artigos, dos quais oito foram excluídos por serem anteriores ao recorte temporal estipulado, seis por serem repetidos da base LILACS e 21 por não responderem à temática. Portanto, nenhum artigo desta biblioteca foi analisado. Desse modo, o corpus desta revisão integrativa foi constituído de 14 artigos, sendo um da BDENF e 13 da LILACS.

Para análise dos estudos, utilizou-se a Análise de Conteúdo proposta por Bardin (2009), que consiste em três etapas: pré-análise, exploração do material e interpretação dos resultados. A primeira e segunda etapas permitiram uma visão abrangente do conteúdo dos artigos por meio de leitura. Posteriormente, na fase de interpretação dos resultados, foram discutidos os resultados com base em diferentes autores (BARDIN, 2009).

No que tange à dimensão ética do estudo, como este é de natureza bibliográfica, não foi necessário o trâmite do projeto para aprovação no Comitê de Ética em Pesquisa (CEP). Entretanto, os preceitos de autoria foram respeitados.

\section{Apresentação e discussão dos resul- tados}

A amostra final foi composta de 14 estudos, sendo a maioria publicada nos anos de 2012 e 2014. No que se refere ao idioma, predominaram os estudos publicados em português $(85,7 \%)$ - dois manuscritos foram publicados em inglês, e nenhum foi publicado em espanhol. Quanto ao periódico de publicação, a Revista Brasileira de Enfermagem abarcou dois estudos (14,3\%).

A partir da análise do conteúdo dos estudos selecionados, emergiram as seguintes categorias: potencialidades da inserção dos profissionais integrantes da Residência Multiprofissional nos serviços; e fragilidades e necessidades para inserção dos profissionais integrantes da Residência Multiprofissional nos serviços de saúde.

3.1 Potencialidades da inserção dos profissionais integrantes da Residência Multiprofissional nos serviços de saúde

A residência proporciona a sistematização do ensino prático, promove a autonomia dos sujeitos e a aproximação do ensino à realidade, formando indivíduos críticos e reflexivos preparados para atuar nas diversas problemáticas que o contexto do seu cotidiano pode oferecer, tornando, assim, a residência menos extenuante, aflitiva e estressante. $\mathrm{O}$ aprendizado adquirido durante a 
residência é reconhecido como fundamental para a formação pessoal e profissional, além de estimular a inserção social de usuários nas atividades educativas, promovendo saúde (MANHO, SOARES, NICOLAU, 2013; MELO, QUELUCI, GOUVÊA, 2014).

Sendo assim, a aprendizagem significativa ocorre por meio da apreensão do conhecimento, em que cada sujeito constrói o seu próprio saber, tendo a problematização como uma importante ferramenta para essa aquisição. É considerado um método instrucional, que visa propiciar ao educando o conhecimento aplicável à solução de problemas, procurando explicar os processos subjacentes, princípios ou mecanismos que sustentam as questões estudadas (BRASIL, 2006).

O ponto mais relevante observado nos vários estudos é, sem dúvida, a atuação diante de uma situação problema, em que se conhece o contexto onde o sujeito está inserido e, a partir dessa realidade, faz-se um planejamento de uma ação por meio de conhecimento de núcleos diferentes com propósitos semelhantes. Existe a necessidade de uma atenção em saúde fortemente baseada nos princípios do SUS, a fim de nortear as ações dos profissionais que constituem esse processo por meio de ferramentas para a aquisição de autonomia.

Um dos dispositivos para a transformação do cuidado é o preparo dos trabalhadores, já que a atualização permite desenvolver a autonomia de quem cuida e de quem é cuidado. A ação produtiva é, assim, duplamente transformadora, porque muda quem realiza o cuidado e quem o recebe (SILVA et al., 2014).

As ações de educação permanente desenvolvidas também pelos residentes permeiam sua formação, tornando o trabalhador um ser reflexivo diante de suas práticas, além de mudar visões paradigmáticas do cuidado e instaurar, dessa forma, a relação positiva da atuação multiprofissional (SILVA et al., 2014). Para que ocorram mudanças de práticas de gestão e de atenção, é imprescindível a capacidade de problematização no trabalho da equipe, sendo, ainda, fundamental que nos tornemos capazes de questionar as concepções vigentes com as ações produzidas e permear novos caminhos de acordo com os princípios que norteiam o SUS (CECCIM, 2005).

No momento em que se reconhece a importância da interdisciplinaridade, estabelecem-se novas óticas de cuidado, gerando um aspecto de integralidade em que as diversas áreas que compõem o grande grupo atuam como facilitadores para a construção de novos paradigmas. Nesse espaço, cada um dos núcleos formadores das Residências Multiprofissionais pode contribuir para que se afirmem como elementos de mudança no fazer saúde, uma vez que o processo de construção da multiprofissionalidade em saúde a partir dessa modalidade de capacitação se apresenta como potente em relação à possibilidade de mudança do modelo assistencial médico centrado (SALVADOR et al., 2011; SANTOS, LANZA, CARVALHO, 2011).

E ainda é importante que o residente e a equipe saibam diferenciar o papel de ambos. O preceptor consiste no supervisor direto das práticas, estando vinculado à instituição formadora ou executadora $\mathrm{e}$ tendo, dentre suas competências, a função de promover essa integração da RMS com usuários. Já a equipe deve proporcionar a aquisição das competências previstas no Projeto Pedagógico do programa, identificando dificuldades e problemas de qualificação do(s) residente(s) relacionados ao desenvolvimento de atividades práticas (BRASIL, 2012).

A receptividade quanto à inserção dos profissionais nos cenários de prática, bem como o entrosamento com os trabalhadores, foi um dos pontos de destaque desta experiência profissional. A fim de que ocorra integração entre a equipe e a RMS, é imprescindível o delineamento no que tange às atribuições do residente e do preceptor, sejam elas relacionadas com conhecimentos con- 
ceituais nos campos da saúde coletiva e das relações humanas ou com conhecimento e desenvoltura na utilização de métodos de ensino (problematização, educação permanente em saúde e educação popular). Além disso, o residente precisa ser capaz de desenvolver relações harmônicas e de participação (PINTO, FORMIGLI, RÊGO, 2007; PAGANI, ANDRADE, 2012).

3.2 Fragilidades e necessidades para inserção dos profissionais integrantes da Residência Multiprofissional nos serviços de saúde

Dentre as fragilidades observadas, uma delas foi a necessidade de maior clareza no que concerne ao papel do interno e aos objetivos do internato para todos os envolvidos, bem como quanto aos problemas relacionados ao apoio material e à supervisão. Há distorções quanto à compreensão dos preceptores e residentes acerca de alguns aspectos que compuseram o processo pedagógico com vistas à aproximação da prática multiprofissional. É necessária, assim, a identificação desses fatores que interferem na inserção dos profissionais nos cenários de atuação para que se possa pensar em novas estratégias e maneiras de inseri-los nos serviços de saúde (PINTO, FORMIGLI, RÊGO, 2007; MANHO, SOARES, NICOLAU, 2013).

Os resultados de alguns estudos revelam que, em um ano e meio do referido programa, os residentes sentiramse instrumentalizados e identificaram dificuldades, como a falta de estrutura organizacional para integração e discussão de problemas. Outros fatores relatados dizem respeito ao afastamento do residente do contexto laboral, à dissociação entre teoria e prática e à inadequação dos procedimentos de supervisão e de avaliação sobre as atividades dos moradores. A competência da gestão é pouco aprofundada em sua formação prévia: ao mesmo tempo que foi considerada bastante potente nos espaços intraequipe e de controle social, apresentou-se quase nula no referente à articulação de rede e macrogestão (GOULART et al., 2012; MANHO, SOARES, NICOLAU, 2013; BRITES et al., 2014; MELO, QUELUCI, GOUVÊA, 2014).

O estudo evidenciou que trabalhar na Estratégia Saúde da Família complexifica o trabalho e que os profissionais vivenciam a dificuldade de compartilhar saberes e de transitar entre o multi e o inter. Conclui-se que a interdisciplinaridade requer uso integrado de conhecimentos na prática multiprofissional, invasão das fronteiras disciplinares, desenvolvimento de competências para lidar com os desafios do meio e atitude individual como componente fundamental para o agir profissional. A interdisciplinaridade e a equipe integrada ainda se apresentam como desafios a serem conquistados (SALVADOR et al., 2011; SCHERER, PIRES, JEAN, 2012).

A exposição aos fatores estressores da profissão e da formação pode favorecer a ocorrência de doenças ocupacionais nesses profissionais, situação que pode ter início no decorrer do curso de graduação. Além disso, foi possível identificar que há residentes, sobretudo adultos jovens, com indicativo para Burnout. Acredita-se que essa formação seja ineficiente para preparar os profissionais para atenderem às demandas do mercado quanto ao significado atribuído à vivência hospitalar. $\mathrm{O}$ mercado exponha esses profissionais a cenários e situações que escapam ao seu arcabouço teórico, exigindo reflexão e julgamento clínico (LANDIM, BATISTA, SILVA, 2010; SALVADOR et al., 2011; GUIDO et al., 2012).

Os conflitos e paradoxos da visão interdisciplinar e do trabalho em equipe ainda precisam de avanços. Ademais, os profissionais da área necessitam ter ampliadas suas bases para enfrentar os dilemas do trabalho em equipe frente a 
uma estrutura hierarquizada. A intrínseca característica da interdisciplinaridade confere caráter inovador aos programas de RMS, demonstrado principalmente por meio da inclusão das 14 categorias profissionais da saúde (BRASIL, 1998).

Ainda que tenham se passado 25 anos da criação do SUS, os discursos quanto à criação da RMS são tanto a favor quanto contra a proposta. No caso destes, incluem-se as produções dos pertencentes à categoria médica, que ocupam espaços de poder e se mostram contrários à proposta da RMS (DALLEGRAVE, KRUSE, 2009; FERREIRA, VARGA, SILVA, 2009).

\section{Considerações finais}

A partir da análise realizada neste estudo, identificaram-se fatores que interferem na inserção dos profissionais integrantes das Residências Multiprofissionais em serviços de saúde. Ademais, os resultados apontam para a necessidade de produções que permitam dar voz aos profissionais dos serviços de saúde no que concerne à inserção da Residência Multiprofissional nesses espaços de atuação.

Acredita-se que, conhecendo a percepção dos profissionais dos serviços, poderão ser elaboradas estratégias de intervenção por meio da interação entre todos os sujeitos envolvidos. Além disso, torna-se necessário ter consonância com as diretrizes do SUS, para proporcionar um atendimento integral e resolutivo ao usuário do sistema.

Pode-se afirmar, também, a necessidade de compreender com mais profundidade os motivos que ocasionam tais dificuldades de enfrentamento em relação ao papel do residente frente ao processo de trabalho. Evidenciou-se, por exemplo, que o preceptor é um dos corresponsáveis pela inserção dos residentes nos campos de atuação, necessitando, assim, de competências para exercer seu trabalho com eficiência.
Os estudos analisados mostraram, ainda, que, quando ocorre a inserção dos profissionais residentes no campo, pelo menos um dos integrantes da equipe apresenta resistência na recepção desse residente, sendo este fator uma limitação e dificuldade encontrada durante a inserção e permanência dos residentes no serviço. Diante do pressuposto e da importância da Residência Multiprofissional para a formação profissional e para o serviço em saúde, é preciso pensar em estratégias para mudar a concepção quanto aos modelos paradigmáticos centrados na figura do biomédico, percebendo a necessidade das demais profissões.

Também se fazem necessários mais estudos que fortaleçam as produções relacionadas aos aspectos potencializados da formação de residentes e a sua possibilidade de transformação do processo de trabalho por meio da implementação da educação permanente nos espaços onde se produz saúde. Ainda, necessário é preciso reconhecer a área do cuidado de enfermagem na educação em saúde e do ensino de profissionais da saúde.

\section{Referências}

BARDIN, L. Análise de conteúdo. Lisboa: Edições; 2009.

BRASIL. Ministério da Saúde. Secretaria de Gestão do Trabalho e da Educação na Saúde. Departamento de Gestão da Educação na Saúde. Residência multiprofissional em saúde: experiências, avanços e desafios / Ministério da Saúde, Secretaria de Gestão do Trabalho e da Educação na Saúde, Departamento de Gestão da Educação em Saúde. - Brasília: Ministério da Saúde, 2006.

BRASIL. Ministério da Saúde. Lei n. 8080/90, de 19 set. 1990. Dispõe sobre as condições para a promoção, proteção e recuperação da saúde, a organização e o funcionamento dos serviços correspondentes e dá outras providências, 
1999. Disponível em: <http://dtr2004. Saude.gov.br/dab/legislacao.php $>$. Acesso em: 03.07.14.

BRASIL. Ministério da Saúde. Lei n. 8142/90, de 28 dez. 1990. Dispõe sobre a participação da comunidade na gestão do Sistema Único de Saúde (SUS) e sobre as transferências intergovernamentais de recursos financeiros na aera da saúde e dá providências, 1990. Disponível em: <http://dtr2004.Saude. gov.br/dab/legislacao.php $>$. Acesso em: 03.07.14.

BRASIL. Ministério da Saúde. Resolução CNRMS $\mathbf{N}^{0}$ 2, de 13 de Abril de 2012. Disponível em <http:portal. mec.gov.br/index.php?option=com_docman\&task $>$. Acesso em 07.09.15.

BRASIL. Ministério da Saúde. Resolução CNS no 287/ 1998 de 08 de outubro de 1998. Disponível em: <http:// bvsms.saude.gov.br/bvs/saudelegis/ cns/1998/res0287_08_10_1998.html $>$. Acesso em: 30.08.15.

BRITES, L. S., WEILLER, T. H., SILVEIRA, D., FRANÇÓIS, A. P. W., ROSA, F. M., RIGHI, L. B. "A gente vai aprendendo": o apoio matricial na estratégia de saúde da família em um programa de residência multiprofissional integrada no interior do Rio Grande do Sul. Brasil. Saúde debate;38(101):295-295, Apr-Jun/2014.

CECCIM, R. B. Educação Permanente em Saúde: desafio ambicioso e necessário. Interface - Comunic, Saúde, Educ, v.9, n.16, p.161-77, set.2004/ fev.2005.

DALLEGRAVE, D., KRUSE, M. H. L. No olho do furacão, na ilha da fantasia: a invenção da residência multiprofissional em saúde. Interface comun. saúde educ;13(28):213-226, jan.-mar. 2009.

FERREIRA, R. C., VARGA, C. R. R., SILVA, R. F. Trabalho em equipe multiprofissional: a perspectiva dos residentes médicos em saúde da família. Ciênc. saúde coletiva;14(supl.1):1421-1428, set.-out. 2009.

GOULART, C. T., SILVA, R. M., BOLZAN, M. E. O., GUIDO, L. A. Perfil sociodemográfico e acadêmico dos residentes multiprofissionais de uma universidade pública. Rev. RENE;13(1):178-186, jan.-fev. 2012.

GUIDO, L. A., SILVA, R. M., GOULART, C. T., BOLZAN, M. E. O., LOPES, L. F. D. Síndrome de Burnout em residentes multiprofissionais de uma universidade pública / Rev. Esc. Enferm. USP;46(6):1477-1483, dez. 2012.

LANDIM, A. S., BATISTA, N. A., SILVA, G. T. R. Vivência clínica hospitalar: significados para enfermeiros residentes em Saúde da Família. Rev. bras. enferm;63(6):913-920, nov.-dez. 2010.

LOBATO, C. P. Formação dos trabalhadores de saúde na residência multiprofissional em saúde da família: uma cartografia da dimensão política. 2010. Tese (Doutorado em Saúde Coletiva)-Universidade Estadual de Londrina, Londrina, 2010.

MANHO, F., SOARES, L. B. T., NICOLAUS, S. M. Reflexões sobre a prática do residente terapeuta ocupacional na estratégia saúde da família no município de São Carlos. Rev. ter. ocup;24(3):233-241, set.-dez. 2013.

MELO, M. C., QUELUCI, G. C., GOUVÊA, M. V. Problematizando a residência multiprofissional em oncologia: protocolo de ensino prático na perspectiva de residentes de enfermagem. Rev. Esc. Enferm. USP;48(4):714714, 08/2014.

MENDES, K. D. S., SILVEIRA, R. C. C. P., GALVÃO, C. M. Revisão integrativa: método de pesquisa para a incorporação de evidências na saúde e na enfermagem. Texto \& contexto enferm (4):758-764, 2008.

PAGANI, R., ANDRADE, L. O. M. Preceptoria de território, novas práticas e saberes na estratégia de educa- 
ção permanente em saúde da família: o estudo do caso de Sobral, CE. Saúde Soc;21(supl.1):94-106, maio 2012.

PINTO, L. L. S., FORMIGLI, V. L. A., RÊGO, R. C. F. A dor e a delícia de aprender com o SUS: integração ensino-serviço na percepção dos internos em medicina social. Rev. baiana saúde pública;31(1):115-133, jan.-jun, 2007.

SALVADOR, A. S., MEDEIROS, C. S., CAVALCANTI, P. B., CARVALHO, R. N. Construindo a Multiprofissionalidade: um Olhar sobre a Residência Multiprofissional em Saúde da Família e Comunidade. Rev. bras. ciênc. saúde;15(3), dez. 2011.

SANTOS, E. R., LANZA, L. M. B., CARVALHO, B. G. Educação permanente em saúde: a experiência do serviço social com Equipes Saúde da Família. Textos contextos (Porto Alegre);10(1):16-25, jan.-jul. 2011.

SCHERER, M. D. A, PIRES, D. E. P., JEAN, R. A construção da interdisciplinaridade no trabalho da Equipe de Saúde da Família. Ciênc. saúde coletiva;18(11):3203-3212, Nov. 2013.set. 2012.

SILVA, C. T., TERRA, M. G., CAMPONOGARA, S., KRUSE, M. H. L., ROSO, C. C., XAVIER, M. S. Educação permanente em saúde a partir de profissionais de uma residência multidisciplinar: estudo de caso. Rev. gaúch. enferm; 35(3):54-54, Sep/2014. 